\title{
Interventional treatment of a symptomatic neonatal hepatic cavernous hemangioma using the Amplatzer vascular plug
}

\author{
Kretschmar, O ; Knirsch, W ; Bernet, V
}

\begin{abstract}
Percutaneous intervention is one treatment option for symptomatic hepatic hemangioma in infants. We report the case of a newborn (birth weight $4060 \mathrm{~g}$ ) with a large hepatic cavernous hemangioma, which presented early with high cardiac output failure due to arteriovenous shunting and signs of incipient Kasabach-Merritt syndrome. We performed a successful superselective transcatheter coil embolization of three feeding arteries on the seventh day of life. Because of remaining diffuse very small arteries causing a relevant residual shunt, additional occlusion of the three main draining veins was necessary with three Amplatzer vascular plugs. Cardiac failure resolved immediately. Without any additional therapy the large venous cavities disappeared within the following months. The tumor continues to regress in size 8 months after the intervention.
\end{abstract}

DOI: https://doi.org/10.1007/s00270-006-0123-7

Posted at the Zurich Open Repository and Archive, University of Zurich ZORA URL: https://doi.org/10.5167/uzh-13262

Journal Article

Published Version

Originally published at:

Kretschmar, O; Knirsch, W; Bernet, V (2008). Interventional treatment of a symptomatic neonatal hepatic cavernous hemangioma using the Amplatzer vascular plug. Cardiovascular and Interventional Radiology, 31(2):411-414.

DOI: https://doi.org/10.1007/s00270-006-0123-7 


\title{
Interventional Treatment of a Symptomatic Neonatal Hepatic Cavernous Hemangioma Using the Amplatzer Vascular Plug
}

\author{
Oliver Kretschmar, ${ }^{1}$ Walter Knirsch, ${ }^{1}$ Vera Bernet $^{2}$ \\ ${ }^{1}$ Division of Pediatric Cardiology, University Children's Hospital Zurich, Steinwiesstrasse 75, 8032 Zurich, Switzerland \\ ${ }^{2}$ Division of Neonatology and Pediatric Intensive Care, University Children's Hospital Zurich, Steinwiesstrasse 75, 8032 Zurich, Switzerland
}

\begin{abstract}
Percutaneous intervention is one treatment option for symptomatic hepatic hemangioma in infants. We report the case of a newborn (birth weight $4060 \mathrm{~g}$ ) with a large hepatic cavernous hemangioma, which presented early with high cardiac output failure due to arteriovenous shunting and signs of incipient Kasabach-Merritt syndrome. We performed a successful superselective transcatheter coil embolization of three feeding arteries on the seventh day of life. Because of remaining diffuse very small arteries causing a relevant residual shunt, additional occlusion of the three main draining veins was necessary with three Amplatzer vascular plugs. Cardiac failure resolved immediately. Without any additional therapy the large venous cavities disappeared within the following months. The tumor continues to regress in size 8 months after the intervention.
\end{abstract}

Key words: Interventional therapy-Neonatal hepatic hemangioma-Vascular plug

Infantile hepatic hemangiomas are frequent. Mostly they are diagnosed within the first 6 months of life. Clinical manifestations can be heart failure due to a relevant left-to-right shunt with tachydyspnea, liver enlargement, consumption coagulopathy with anemia and thrombopenia (Kasabach-Merritt syndrome), and cutaneous hemangioma [1-3]. The diagnostic modalities commonly used are ultrasonography, with computed tomography (CT) or magnetic resonance imaging (MRI). Treatment strategies include medical and surgical options, as well as an interventional approach, and depend on tumor size, growth characteristics, location, and complications [1-8]. Established medical treatment consists of steroids and interferon-alpha [1, 2, 4, 5]. Surgically, arterial feeding vessels may be ligated, hemangiomas can be resected and, in severe cases, liver transplantation is possible [1, 2, 3, 9].

In symptomatic patients, percutaneous catheter embolization has been increasingly performed in recent years due to good experiences, good results, and optimized endovascular techniques

Correspondence to: Oliver Kretschmar; email: oliver.kretschmar@kispi. unizh.ch
[5-8]. Until now, in all reported cases of interventional treatment, only embolizations of the arterial feeders, either with coils [5-7] or with glue [8], have been described.

We report the case of a symptomatic male newborn with a large cavernous hepatic hemangioma. He was admitted in high-output cardiac failure due to the arteriovenous shunt with signs of incipient Kasabach-Merritt syndrome. Coil embolization of the main arterial feeders was not sufficient because of diffuse additional smaller arterial vessels. To our knowledge, the additional successful venous occlusion of the main draining veins in a newborn has not previously been described. Furthermore, the Amplatzer vascular plug (AGA Medical, Golden Valley, MN, USA), which has recently been developed for closure of vascular abnormalities [10], was used in this case for the first time for venous occlusion of a hepatic hemangioma in a newborn.

\section{Case Report}

A newborn male at 40 weeks of gestation with a birth weight of $4060 \mathrm{~g}$ developed unexplained mild cyanosis, massive edema especially in the upper part of the body, and severe hypoglycemia during the first day of life. Ultrasonography revealed a large mass of $6 \mathrm{~cm} \times 6 \mathrm{~cm} \times 6 \mathrm{~cm}$ within the lower part of the right liver lobe with the appearance of a cavernous hemangioma. The draining liver veins as well as the inferior caval vein were extensively dilated. Radiography (Fig. 1(1)) showed a global enlargement of the heart and dilatation of the central pulmonary arteries. Subsequent echocardiography confirmed mild heart failure with right atrial and ventricular dilatation, slightly reduced ventricular contractility, and intermittent right-to-left shunting through a patent foramen ovale from volume overload. Despite anticongestive therapy, tachypnea and cardiac enlargement persisted. Furthermore the patient underwent intermittent phototherapy for recurrent hyperbilirubinemia. MRI of the abdomen clearly showed the large singular cavernous hemangioma in the right lower lobe of the liver with dilatation of all liver veins (Fig. 2). Persisting cardiac failure despite therapy and thrombocytopenia, which was interpreted as the signs of incipient Kasabach-Merritt syndrome, motivated interventional catheterization on the seventh day of life.

Catheterization took place with the patient under general anesthesia using a $4 \mathrm{Fr}$ sheath in the right femoral artery, and initially also a $4 \mathrm{Fr}$ sheath in the right femoral vein. Cefazolin was administered three times within the first $24 \mathrm{hr}$, starting with the first dose during the procedure. Standard right and left heart catheterization revealed the main pulmonary artery pressure to be elevated to $38 / 16 / 25 \mathrm{mmHg}$, with a calculated pulmonary-to-systemic flow ratio of 7.0. The descending aortogram 

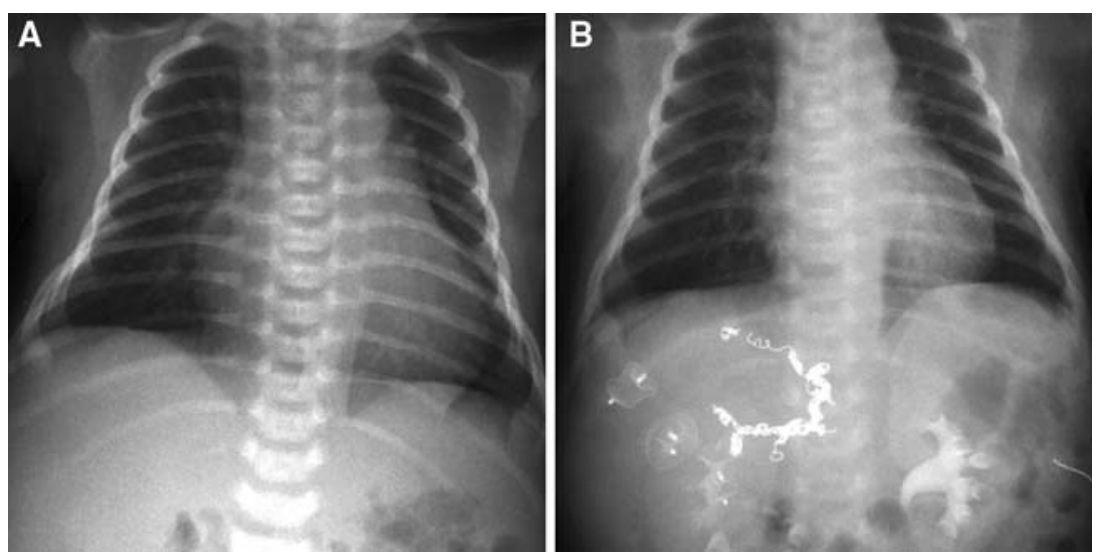

Fig. 1. Chest X-ray (A) Cardiac and hepatic enlargement before intervention. (B) Normalization of cardiac enlargement 1 day after intervention. Platinum embolization coils in arteries supplying the hepatic hemangioma, mainly arising from the coeliacal trunc, and 3 Amplatzer Vascular Plugs, with a size of 8,10 and $12 \mathrm{~mm}$, respectively, in the 3 main draining intrahepatic vessels.

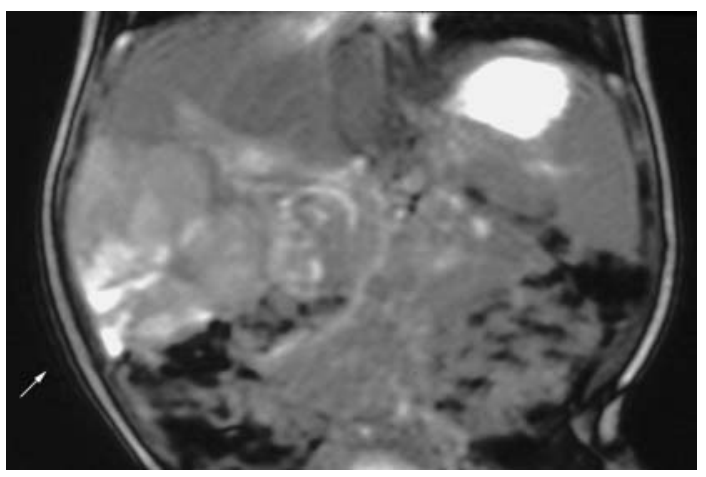

Fig. 2. MRI of the abdomen demonstrating the large singular cavernous hemangioma in the right lower lobe of the liver with dilatation of all liver veins.

demonstrated the arterial feeding vessels of the hepatic hemangioma, arising mainly from the celiac trunk with additional diffuse small arteries from the intercostal arteries (Fig. 3). The three main feeders were reached with a guiding 4 Fr Right Coronary Amplatz Modified Super Torque catheter (Cordis, Johnson \& Johnson, Miami, FL, USA), and selectively with a VASCO + 18 microcatheter (BALT, Montmorency, France). They were uneventfully supraselectively embolized with 15 platinum embolization pushable coils (BALT, Montmorency, France) of different sizes (2 to $3 \mathrm{~mm}$ ) and lengths $(50$ to $80 \mathrm{~mm})$. A repeat angiogram demonstrated the complete closure of these vessels, but the remaining smaller and diffuse arteries from the intercostal arteries still provided a significant source of blood supply to the hepatic hemangioma. It was not possible to close them because of their diffuse origins and small vessel diameters.

Because of the insufficient result, an additional venous intervention was considered. Therefore, the $4 \mathrm{Fr}$ sheath in the femoral vein was exchanged for a 6 Fr Flexor Check-Flo Introducer sheath (Cook Medical, Bloomington, IN, USA), which was placed in the right upper liver vein. Selective angiography showed the cavernous venous part of the hemangioma and three main draining veins from the dilated liver veins (Fig. 4). It was possible to reach each draining vein near its origin with the guiding catheter, and to implant an $8 \mathrm{~mm}, 10 \mathrm{~mm}$, and $12 \mathrm{~mm}$ Amplatzer Vascular Plug, respectively. The plugs were attached to a nitinol pusher delivery cable. The transfer through the sheath was easy, and the plugs even tracked perfectly around the proximal sharp sheath curve $\left(90-120^{\circ}\right)$. After a test injection through the sheath, which confirmed the excellent configuration and position of each device, the device was released by unscrewing the delivery cable. Final arterial and venous angiograms demonstrated clearly reduced arterial and venous flow through the hem-

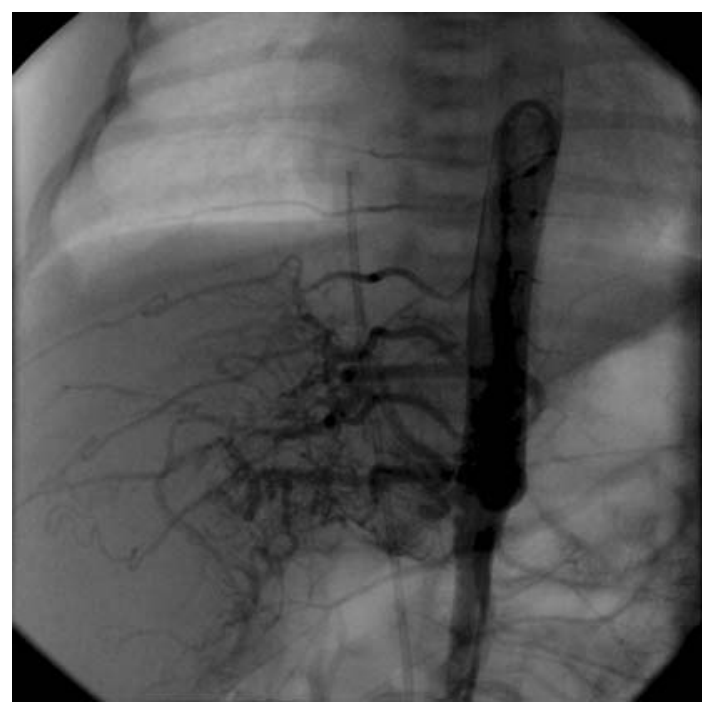

Fig. 3. An angiogram in the descending thoracic/abdominal aorta demonstrating multiple arterial feeding vessels of the hepatic hemangioma arising mainly from the celiac trunc and additionally with diffuse small arteries from intercostal arteries.

angioma with slowed, but not completely interrupted, venous flow through the wire mesh of the vascular plugs (Fig. 5). The pulmonary-tosystemic flow ratio was diminished to 1.4. No procedure-related complication occurred.

The patient improved quickly after the embolization, and was extubated 1 day after the intervention. Radiography demonstrated an immediate reduction in size of the heart (Fig. 1 (2)). Pretreatment cardiac dilatation and heart failure disappeared quickly. The serum aminotransferase levels, which were initially elevated up to $1000 \mathrm{IU} / \mathrm{l}$, normalized within days. Platelet count and liver function improved, and no substitution of platelets or fresh frozen plasma was necessary. Because of this good post-interventional result, treatment with corticosteroids or interferon-alpha was not considered necessary for adjunctive therapy. The patient was discharged home 12 days after the intervention with mild anticongestive treatment (diuretics), and oral antiplatelet treatment with aspirin. Diuretics and aspirin were continued for 12 weeks. Abdominal ultrasonography showed immediate regression of the intrahepatic lesion. After 3 months, the cavernous structures of the hemangioma were no longer detectable and the venous Amplatzer plugs did not show any residual flow. Neurologic development was normal, and there were no residual signs of heart failure over a followup period of 8 months. 


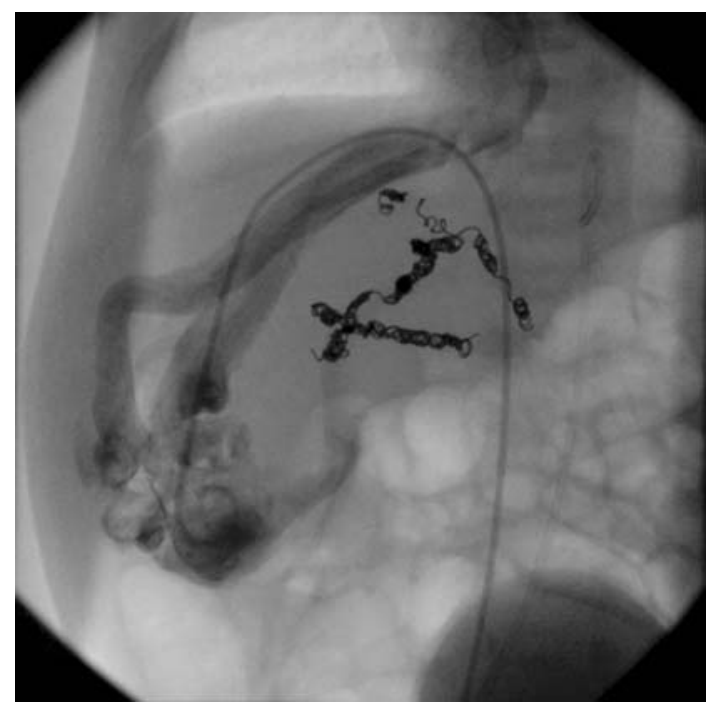

Fig. 4. A selective angiogram of the cavernous part of the hemangioma showing three main draining veins into the dilated liver veins. Multiple embolization coils in the feeding arteries.

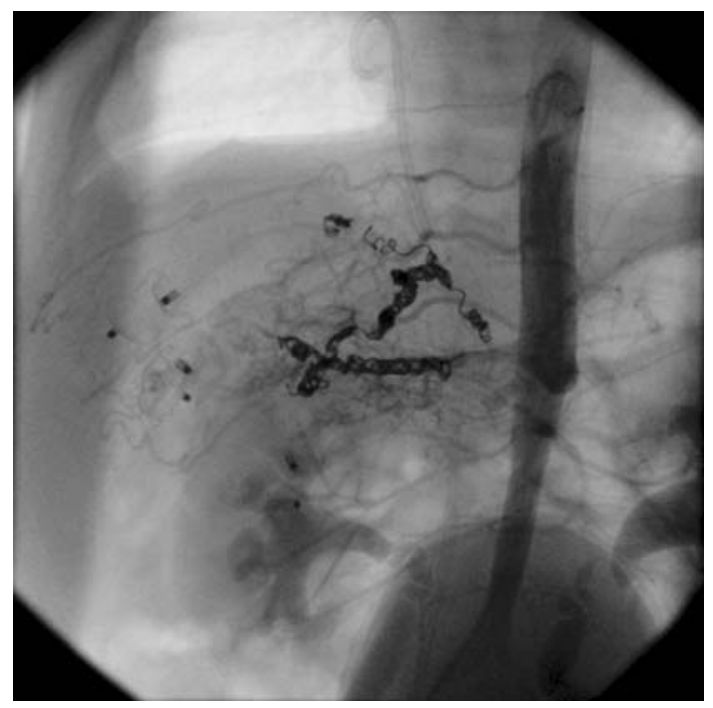

Fig. 5. A final angiogram in the descending aorta demonstrating a clearly reduced arterial and venous flow through the hemangioma and demonstrating the correct position and configuration of the implanted coils and Amplatzer Vascular Plugs.

\section{Discussion}

Neonatal intrahepatic hemangioma and arteriovenous malformations can cause early congestive heart failure by severe arteriovenous shunting. In the past, these patients have had to be treated surgically $[1,2,3,9]$ by partial hepatectomy, ligation of the hepatic artery, or even orthotopic liver transplantation in the neonatal period. A developing alternative in these cases is therapeutic selective interventional hepatic embolization of feeding arteries [1, 3, 5-8].
In asymptomatic patients, even a conservative approach without any therapy can be considered because of the high possibility of spontaneous regression within the first year of life [3].

There already exists good evidence from smaller patient groups or case reports that drug therapy, alone or together with surgery, or intervention with corticosteroids and/or interferon-alpha may efficiently reduce the volume of a hemangioma $[1-5,7,9]$.

The results of interventional therapy in patients in whom medical therapy has failed or the symptoms of cardiac failure are rapidly progressive, are mainly encouraging. Symptoms leading to an interventional approach are heart failure, consumptive coagulopathy (Kasabach-Merritt syndrome), anemia, abdominal mass, and respiratory distress requiring mechanical ventilation $[1,3,5-$ 8]. Selective embolization of the feeding arteries can be achieved using small flexible target or detachable coils [1, 3, 5-7]. The use of a mixture of lipiodol and Histoacryl glue for smaller arteries has been described with good results in 2 patients [8]. Sometimes even after an obviously successful embolization, "residual" arterial perfusion of the tumor can be shown immediately after coil occlusion. The vascular supply may arise not only from hepatic artery branches, but also from extrahepatic collaterals from the superior mesenteric artery, intercostals, phrenic and adrenal arteries, and from portal veins [7, 8]. If these residual collaterals do not cause further hyperperfusion with congestive heart failure, there is no need to occlude them, because they will probably regress within months [7]. Otherwise, the tumor may also be surgically resected after cardiopulmonary stabilization, allowing improved operability.

Our patient presented with signs of cardiac failure due to arteriovenous shunting (pulmonary-to-systemic flow ratio of 7.0), the signs of incipient consumptive coagulopathy, and liver insufficiency. Therefore, the interventional approach was indicated. We also saw a multifocal vascular supply to the cavernous hemangioma. Though the main feeding arteries arising from the hepatic artery could be selectively coil embolized, the remaining smaller collaterals, mainly from intercostal arteries, were responsible for continuing and hemodynamically relevant arteriovenous shunting. An additional venous approach with occlusion of the main draining intrahepatic veins near their origins from the tumor was successfully performed with three Amplatzer vascular plugs. Cardiac failure resolved immediately within the first days after the intervention, and the intrahepatic lesion continued to regress.

We used the Amplatzer vascular plug for this novel approach both because it is a new device specific for vessel occlusion, and for its extremely user-friendly simple mechanics. The selfexpandable cylindrical nitinol device is attached to a flexible nitinol delivery cable and comes preloaded in a loader. In case of unsatisfactory positioning, the device can easily be retrieved with this cable. As soon as the position is verified, the device can be deployed by unscrewing it. With the above-mentioned characteristics, its low profile, and an acceptable cost-effectiveness, the plug has already been used to occlude most abnormal vessels [10]. Especially in aortico-pulmonary $[10,11]$ and veno-venous $[10,12]$ collaterals, and in pulmonary arteriovenous fistulas [10, 13], it has proven to be a suitable occlusion device. Hill et al. also describe successful closure of a transhepatic access for cardiac catheterization in 7 patients with the Amplatzer vascular plug [10]. For the closure of a patent ductus arteriosus, which has been carried out in 4 patients [10,14], the larger pores of the larger devices may be responsible for a residual shunt [10]. 
In our case we needed a 6 Fr guiding sheath to implant the vascular plugs of $8 \mathrm{~mm}, 10 \mathrm{~mm}$, and $12 \mathrm{~mm}$ diameter. All devices tracked easily around the proximal sharp sheath curve $\left(90-120^{\circ}\right)$ into their final intrahepatic position. Follow-up Doppler studies did not show any occlusion of the femoral vessels. Following the procedure, aspirin was used as an antiplatelet agent at a dose of 4 $\mathrm{mg} / \mathrm{kg}$ per day for 3 months. This might be responsible for the open femoral vessels, but it also probably delayed the complete closure of the vascular plugs. Perhaps a shorter antiplatelet treatment period of 4 weeks would have been sufficient.

To our knowledge this is the first description of an additional venous closure in a symptomatic neonatal intrahepatic hemangioma, as well as the first use of the Amplatzer vascular plug for this condition.

As illustrated in the case, this combined interventional arterial and venous approach can be a successful treatment option where there is multifocal vessel supply to a symptomatic hepatic hemangioma, even in a newborn.

\section{References}

1. Boon LM, Burrows PE, Paltiel HJ, et al. (1996) Hepatic vascular anomalies in infancy: A twenty-seven-year experience. J Pediatr 129:346-354

2. Daller JA, Bueno J, Gutierrez J, et al. (1999) Hepatic hemangioendothelioma: Clinical experience and management strategy. J Pediatr Surg 34:98-105
3. Iyer CP, Stanley P, Mahour GH (1996) Hepatic hemangiomas in infants and children: A review of 30 cases. Am Surg 62:356-360

4. Woltering MC, Robben S, Egeler RM (1997) Hepatic hemangioendothelioma of infancy: Treatment with interferon $\alpha$. J Pediatr Gastroenterol Nutr 24:348-351

5. Fok TF, Chan MS, Metreweli C, et al. (1996) Hepatic hemangioendothelioma with early heart failure in a newborn: Treatment with hepatic artery embolization and interferon. Acta Paediatr 85:1373-1375

6. Peuster M, Windhagen-Mahnert B, Fink C, et al. (1998) Interventional therapy of a hepatic hemangioendothelioma in a newborn using a venous approach. Z Kardiol 87:832-836

7. Warmann S, Bertram H, Kardorff R, et al. (2003) Interventional treatment of infantile hepatic hemangioendothelioma. J Pediatr Surg 38:1177-1181

8. Kullendorff CM, Cwikiel W, Sandstrom S (2002) Embolization of hepatic hemangiomas in infants. Eur J Pediatr Surg 12:348-352

9. Samuel M, Spitz L (1995) Infantile hepatic hemangiothelioma: The role of surgery. J Pediatr Surg 30:1425-1429

10. Hill SL, Hijazi ZM, Hellenbrand WE, et al. (2006) Evaluation of the Amplatzer vascular plug for embolization of peripheral vascular malformations associated with congenital heart disease. Catheter Cardiovasc Interv 67:113-119

11. Hijazi ZM (2004) New device for percutaneous closure of aortopulmonary collaterals. Catheter Cardiovasc Interv 63:482-485

12. De Groote K, Verhaaren H, Suys B, et al. (2005) Percutaneous closure by a vascular plug of a fistula between the superior caval vein and the left atrium. Pediatr Cardiol 26:675-676

13. Farra H, Balzer DT (2005) Trancatheter occlusion of a large pulmonary arteriovenous malformation using the Amplatzer vascular plug. Pediatr Cardiol 26:683-685

14. Hoyer MH (2005) Novel use of the Amplatzer plug for closure of a patent ductus arteriosus. Catheter Cardiovasc Interv 65:577-580 